\title{
Tafamidis Meglumine
}

National Cancer Institute

\section{Source}

National Cancer Institute. Tafamidis Meg/umine. NCI Thesaurus. Code C84194.

A soft gelatin capsule formulation containing the meglumine salt form of tafamidis, a small molecule and pharmacological chaperone with potential disease-modifying activity. T afamidis binds to and stabilizes wild-type and variant (V122I) transthyretin (TTR), thereby preventing tetramer dissociation into monomers; this prevents misfolding of the TTR protein and inhibits the formation of TTR amyloid fibrils and the subsequent deposition of these insoluble protein clusters in peripheral nerve tissues and organs. TTR is a 127 amino acid transport protein for thyroxine and retinol and is secreted by the liver. 